Research Paper

\title{
Multi drug resistance in strong biofilm forming clinical isolates of Staphylococcus epidermidis
}

\author{
Gulcan Sahal, Isil Seyis Bilkay \\ Department of Biology, Faculty of Science, Hacettepe University, Beytepe, Ankara, Turkey.
}

Submitted: February 13, 2013; Approved: September 9, 2013.

\begin{abstract}
Staphylococcus epidermidis which exists in healthy human skin as a commensal inhabitant is also an important pathogen forming biofilms on many surfaces and recently, increased resistance traits were suggested to be acquired in biofilm environments. In this study; clinical Prevalences, antibiotic resistances and biofilm formations of S. epidermidis strains were determined and comparison of all these findings with each other was carried out in order to take precautions against them and figure out if high biofilm forming S. epidermidis strains display multi drug resistance. According to our results; samples of wound and blood were the most S. epidermidis isolated clinical materials $(40 \% ; 35 \%)$ and cardiothoracic surgery was the most $S$. epidermidis observed service unit. All of these strains were sensitive to vancomycin, however $65 \%$ of them showed resistance to all $\beta$-lactam antibiotics (Penicillin, Oxacillin, Amoxicilin / Clavulonic acid), used in this study and $60 \%$ of all S. epidermidis strains were found as multi drug resistant. When the results of strong biofilm forming S. epidermidis strains are examined; they were isolated from sample of blood and service unit of cardiovascular surgery in highest frequency and $80 \%$ of them were $\beta$-lactam resistant whereas $100 \%$ of them were multi drug resistant. One of these multi drug resistant strains which was resistant to maximum amount of different antimicrobial classes, was also observed as maximum biofilm forming strain among all the other S. epidermidis isolates. Multi drug resistance in strong biofilm forming strains shows that; biofilms play a role in antimicrobial resistance traits of $S$. epidermidis.
\end{abstract}

Key words: Staphylococcus epidermidis, biofilm formation, $\beta$-lactam resistance, multi drug resistance, indwelling medical device related infections.

\section{Introduction}

S. epidermidis which is known as a natural colonizer of healthy human skin and mucosa, is also a common nosocomial pathogen along with other Coagulase Negative Staphylococci (CNS) (Otto, 2009) and among them; S. epidermidis is the most important pathogen, responsible from many indwelling medical device related infections such as; catather, prosthetic joint, vascular graft, surgical site, central nervous system shunt and cardiac device related ones. Therefore, S. epidermidis strains especially emerge as life-threatening pathogens triggering septicaemia, meningitis and other serious conditions in medical device using and immunocomprised patients (Goldman et al., 2001; Guiot et al., 1994; Poutsiaka et al., 2007; Ziebuhr et al., 2006). One of the most important mechanisms making these commensal inhabitants dangerous for medical device using and immunocomprised patients is known as biofilm formation. By means of having ability to adhere to various surfaces and form slimy layer known as biofilm on them; $S$. epidermidis strains and some other biofilm forming microorganisms can encase themselves (Costerton et al., 1995; Costerton, 1999; Shiro et al., 1994; Tunney et al., 1998). Thus; neither immune defense nor antimicrobial agents are able to eradicate biofilm-associated infections and in order to terminate them, the device must be removed or some surgical operations must be applied (Xu and Siedlecki, 2012). Moreover, since S. epidermidis strains are natural colonizers of healthy human skin and can easily grow on medical devices because of having strong attachment ability, they

Send correspondence to G. Sahal. Department of Biology, Faculty of Science, Hacettepe University, Beytepe, Ankara, Turkey. E-mail: gozbakir@hacettepe.edu.tr. 
can easily be transmitted to a medical device and colonize on the surface of it during an implantation process (Kaplan et al., 2004). That is, a natural colonizer of healthy human skin may easily turn into a nosocomial pathogen if it has strong attachment and high biofilm forming abilities. Nowadays, increased antibiotic resistance in some clinical strains is thought to be related with their biofilm forming capabilities. Because, acquirement of some resistance traits via gene transfer is possibble within biofilms (Subramanian et al., 2012). The aim of this study is to determine the clinical prevelances, antibiotic resistances and biofilm formations of S. epidermidis strains; compare all these findings with each other, reveal the clinical informations of multi drug resistant and high biofilm forming S. epidermidis strains in order to take precautions against them and figure out if high biofilm forming S. epidermidis strains display multi drug resistance.

\section{Materials and Methods}

\section{Bacterial strains}

All S. epidermidis strains were obtained from a hospital in Ankara, Turkey and were isolated from clinical materials of blood, wound, nose, abscess, synovial fluid and from the services of physical rehabilitation, cardiothoracic surgery, dermatology, intensive care, neurology, general surgery, emergency, thoracic medicine and paediatry. Isolated S. epidermidis strains were inoculated in to the Brain Heart Infusion Broth media including 10\% glycerol and stored at $-20^{\circ} \mathrm{C}$.

Isolates were identified by standard phenotypical methods (Holt et al., 1994) and identification was further confirmed by using Vitek-32 system (BioMérieux, France).

\section{Antibacterial susceptibility testing}

The susceptibilities of S. epidermidis strains to 11 different antibacterial agents of Amoxicilin/Clavulonic acid $(20 / 10 \mu \mathrm{g})$, Erythromycin $(15 \mu \mathrm{g})$, Oxacillin $(1 \mu \mathrm{g})$, Ciprofloxacin $(5 \mu \mathrm{g})$, Trimethoprim/Sulfamethoxazole $(1.25 \mu \mathrm{g})$, Vancomycin $(30 \mu \mathrm{g})$ Gentamicin $(10 \mu \mathrm{g})$, Tetracycline (30 $\mu \mathrm{g})$, Clindamycin $(2 \mu \mathrm{g})$, Penicillin (10 Units), Nitrofurantoin $(300 \mu \mathrm{g})$ were assessed by Disc-Diffusion method according to National Committee for Clinical Laboratory Standards (NCCLS) and the strains were classified as Resistant (R), Intermediate (I) or Sensitive (S), according to the zone table, constituted by Clinical and Laboratory Standards Institute (CLSI).

\section{Biofilm formation}

The determination of biofilm formation in $S$. epidermidis strains was performed by Crystal Violet Binding Assay described by O'Toole with some modifications (O'Toole, 2011). Briefly, bacterial cells corresponding to a 2.0 McFarland optical density standard were inoculated into Brain Heart Infusion Broth medium and then they were incubated at $37{ }^{\circ} \mathrm{C}$ overnight. The overnight culture was 1:100 diluted into a fresh BHI medium and the wells of a polysytrene plate were filled with $1 \mathrm{~mL}$ of the diluted inoculum. Then, the plates were incubated for $48 \mathrm{~h}$ at $37^{\circ} \mathrm{C}$. Following this, the medium was gently removed and the wells were washed. After allowing wells to dry, each of them were stained with $1 \%$ crystal violet for $45 \mathrm{~min}$ at room temperature. Afterwards, the unbound crystal violet stain was removed and microscobic observation was done. Finally, bound crystal violet in each well was solubilized by adding $1 \mathrm{~mL}$ of Ethanol-Acetic Acid (90:10) solution and solubilized crystal violet for each well was read by a spectrofotometer at $540 \mathrm{~nm}$. According to their biofilm formations, S. epidermidis strains were classified into four categories as follows:

$$
\begin{aligned}
& \mathrm{OD}=0, \text { Non Biofilm Former (NBF) } \\
& 0<\mathrm{OD}<0.4 \text {, Weak Biofilm Former (WBF) } \\
& 0.4 \leq \mathrm{OD}<0.8 \text {, Intermediate Biofilm Former (IBF) } \\
& \mathrm{OD} \geq 0.8, \text { Strong Biofilm Former (SBF) } \\
& \text { The experiment was performed in triplicate. }
\end{aligned}
$$

\section{Results}

S. epidermidis strains were obtained from 5 different clinical materials including; blood, wound, nose, abscess and synovial fluid. Among them; samples of wound and blood were the most $S$. epidermidis isolated clinical materials $(40 \% ; 35 \%)$ and among all the service units which include patients infected with S. epidermidis; cardiothoracic surgery was observed in highest frequency (35\%) (Figures 1,2 ).

After the application of Crystal Violet Binding Method for biofilm screening; $25 \%$ of all S. epidermidis strains were determined as strong, $15 \%$ as intermediate, $55 \%$ as weak and $5 \%$ as non-biofilm formers. When the biofilm formations of $S$. epidermidis isolates in different clinical materials are examined, strong biofilm forming strains were isolated from samples of blood, wound and synovial fluid and within these; sample of blood was seen in highest frequency (Figure 1). When the biofilm formations of S. epidermidis strains in different service units are examined, cardiothoracic surgery was the most frequent unit for strong biofilm forming S. epidermidis isolation and additionally, the other units including patients infected with strong biofilm forming $S$. epidermidis strains were observed as; thoracic medicine, neurology and dermatology (Figure 2).

When the antibiogram tests of $S$. epidermidis strains against 11 different antibiotics are examined; all of them were found as sensitive to vancomycin (Figure 3). However, $65 \%$ of them and $80 \%$ of strong biofilm formers showed resistance to all $\beta$-lactam antibiotics (P, OX, AMC) used in this study (Table 1). 


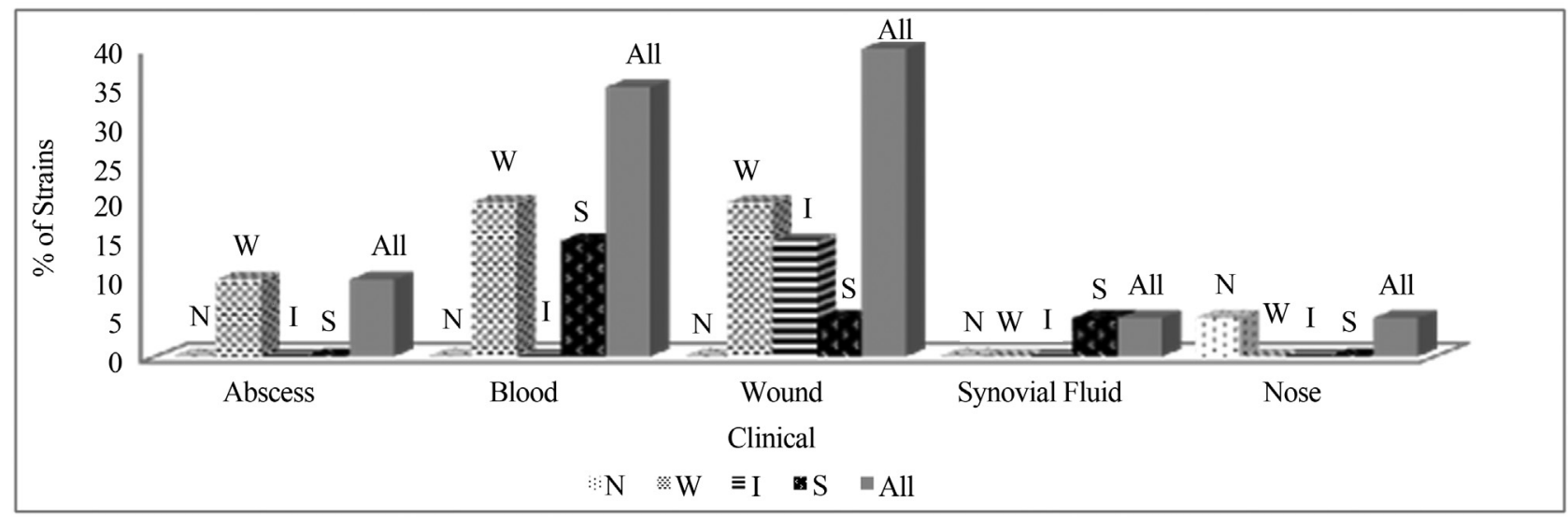

Figure 1 - Percentage of S. epidermidis strains in different clinical materials according to their biofilm formation levels. (N: Non Biofilm Formers, W: Weak Biofilm Formers, I: Intermediate Biofilm Formers, S: Strong Biofilm Formers).

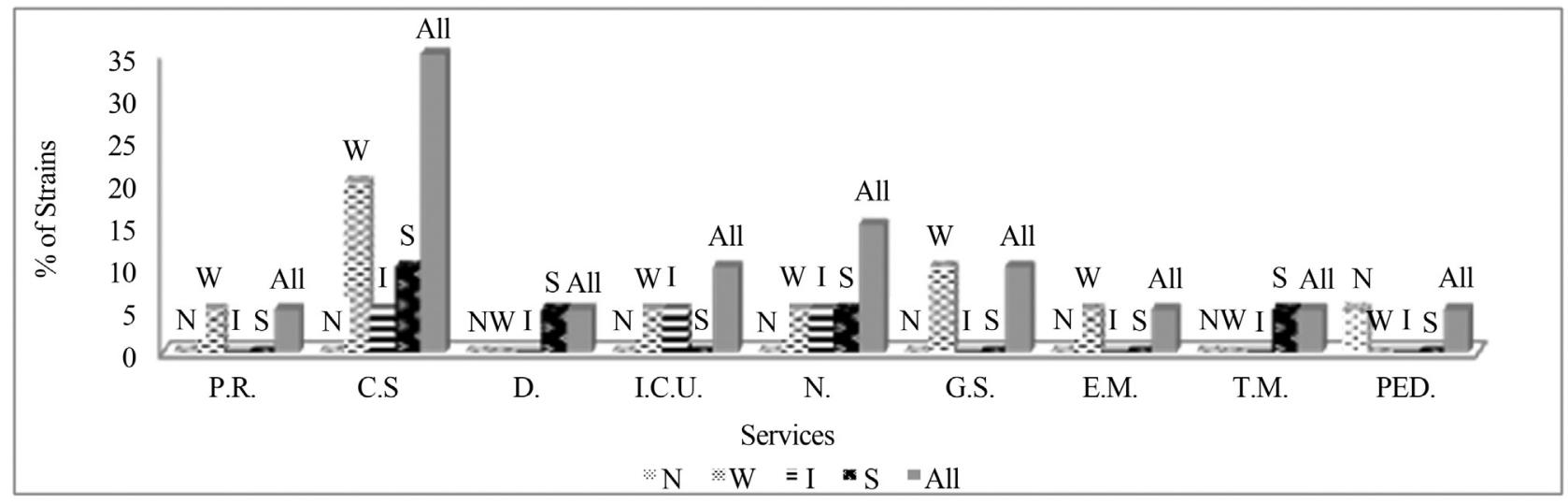

Figure 2 - Percentage of S. epidermidis strains in different service units according to their biofilm formation levels. (P.R.: Physical Rehabilitation, C.S.: Cardiothoracic Surgery, D.: Dermatology, I.C.U.: Intensive Care Unit, N: Neurology, G.S.: General Surgery, E.M.: Emergency, T.M.: Thoracic Medicine, PED: Pediatrics, N: Non Biofilm Formers, W: Weak Biofilm Formers, I: Intermediate Biofilm Formers, S: Strong Biofilm Formers).

In the last part of this study; multi drug resistant strains were determined and their biofilm formations were examined. According to results; $60 \%$ of all S. epidermidis strains and each of strong biofilm forming S. epidermidis strains were found as resistant to 3 or more antimicrobial classes and defined as multi drug resistant (Table 1). In addition; maximum biofilm forming isolate was found as resistant to 7 different (Aminoglycoside, $\beta$-lactams, Macrolide, Tetracycline, Fluoroquinolone, Lincosamide and Sulfonamide) antimicrobial classes.

\section{Discussion}

Technological developments have provided several benefits for human life, both in industry and medical fields. Eradication of pathogen microorganisms by antimicrobial agents and usage of many indwelling medical devices in order to increase the function of any defective organ, are some significant advances which can be given as examples to these benefits (Aybar et al., 2012). Inspite of all these beneficial things, increase in antimicrobial resistant micro- organisms rises every passing day and as a consequence; persistent nosocomial infections in hospitals, mortality and huge economic damages come true. Therefore, investigation of mechanisms playing a role in drug resistance and fighting against them, have great place in preventing these casualties. One of these mechanisms playing a role in persistent infections is known as biofilm formation (Djeribi et al., 2012). Therefore, this study is carried out to reveal the occurrences of $S$. epidermidis strains in different clinical materials and service units, determine antibiotic resistances of them and evaluate all these results according to $S$. epidermidis strains' biofilm formation performances.

When the occurrence of $S$. epidermidis strains according to clinical information of patients from which they were isolated are examined, it is seen that our findings are in agreement with a recent study, suggesting; samples of blood and wound are the most S. epidermidis isolated clinical materials (Sheikh and Mehdinejad, 2012). In addition, according to some other studies; similar to our results, $S$. epidermidis is indicated as the most important pathogen playing a role in cardiovascular infections (Michelim et al., 


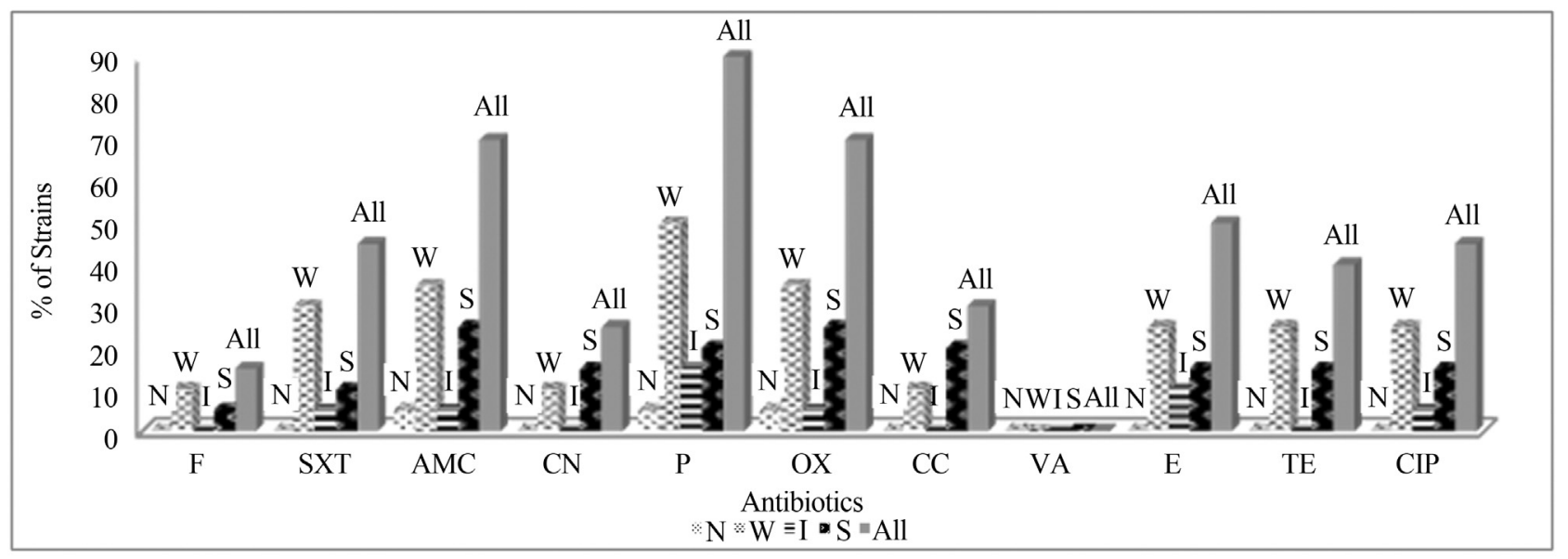

Figure 3 - Percentage of S. epidermidis strains displaying resistance to 11 different antibiotics according to their biofilm formation levels. (F: Nitrofurantoin, SXT: Trimethoprim/Sulfamethoxazole, AMC: Amoxicilin/Clavulonic acid, CN: Gentamicin, P: Penicillin, OX: Oxacillin, CC: Clindamycin; VA: Vancomycin, E: Eritromycin, TE: Tetracyclin, CIP: Ciprofloxacin, N: Non Biofilm Formers, W: Weak Biofilm Formers, I: Intermediate Biofilm Formers, S: Strong Biofilm Formers).

2005; Villari et al., 2000; Vuong and Otto, 2002). Furthermore, Staphylococci are stated as the most commonly diagnosed microorganisms in microbial infections on blood-contacting devices (Xu and Siedlecki, 2012). Existence of high proportion of $S$. epidermidis strains in cardiovascular infections and in sample of blood confirms that, $S$. epidermidis is one of the most important pathogens causing bloodstream infections and the reason of this is suggested to be associated with their biofilm formation abilities on implanted medical devices like prosthetic heart valves (Xu and Siedlecki, 2012).

According to their biofilm formation performances, $S$. epidermidis strains were classified in different groups. Within these, percentage of strong biofilm forming strains were found as $25 \%$. Studies, which have been applied for several years indicate that; usage of intravascular devices in different types, is the reason of most nosocomial blood stream infections (Fux et al., 2003) and the increased usage of these indwelling medical devices such as; cardiac devices, vascular grafts and intravascular catheters, is observed to enhance frequency of biofilm related $S$. epidermidis infections (Arciola et al., 2012). Therefore, it is concluded that; the existence of strong biofilm forming strains in different cardiothoracic surgery unit patients and in sample of blood may be related with enhanced usage of some indwelling medical devices.

When the antibiotic test results of S. epidermidis strains are examined; our findings are in agreement with those of other studies suggesting; vancomycin is the most efficient antibiotic for $S$. epidermidis inhibition (Al et al., 2005; Chaieb et al., 2005; McCann et al., 2008). However; deficient inhibition effect of vancomycin in biomaterial re-

Table 1 - Percentage of Strong Biofilm Forming and all S. epidermidis strains displaying resistance to different antimicrobial classes.

\begin{tabular}{lcc}
\hline Resistance to antimicrobial classes & \% of SBF S. epidermidis strains & \% of All S. epidermidis strains \\
\hline Fully Sensitive & - & 5 \\
Resistance to 1 Antimicrobial Class & - & 15 \\
Resistance to 2 Antimicrobial Classes & 20 & 20 \\
Resistance to 3 Antimicrobial Classes & 20 & 10 \\
Resistance to 4 Antimicrobial Classes & 40 & 15 \\
Resistance to 5 Antimicrobial Classes & - & 20 \\
Resistance to 6 Antimicrobial Classes & 20 & 5 \\
Resistance to 7 Antimicrobial Classes & - & 10 \\
Resistance to 8 Antimicrobial Classes & - & - \\
Resistance to 9 Antimicrobial Classes & 80 & - \\
$\beta$-lactam (OX, AMC, P) Resistance & 100 & 65 \\
*Multi Drug Resistance & & 60 \\
\hline
\end{tabular}

(*Each of Strong Biofilm Forming strains were multi drug resistant).

(Antimicrobial Classes: Aminoglycoside, $\beta$-lactam, Macrolide, Tetracycline, Fluoroquinolone, Lincosamide, Furan, Sulfonamide, Glycopeptide). 
lated endocarditis infections is also pointed out and the reason of this is indicated to be related with biofilm formation (Juárez-Verdayes et al., 2006). When the microorganisms spread to external surface of a medical device, they begin to grow and multiply to colonize on it and in order to fasten their colonization, they produce extracellular polymeric substances known as biofilms (Kumar and Anand, 1998). As a result of including extracellular polymeric substance, biofilms act as preventive barriers both to antimicrobial agents and immunological responses. For this reason, in order to eradicate biofilm related infections, some surgical operations are required to be applied (Baillie and Douglas, 2000; Xu and Siedlecki, 2012). Apart from this, inside biofilms; huge amount of extracellular DNA exists and too many distinct microorganisms have a chance to come together and stay immobile. As a result, in addition to acting as a preventive barrier; biofilm environments also promote genetic exchange of antimicrobial resistance genes, increasing bacterial virulence and contributing to the development of multi resistant phenotypes (de Araujo et al., 2006; Parsek and Singh, 2003; Subramanian et al., 2012). Confirming these, in our study; high amount of $\beta$-lactam resistance was observed in strong biofilm forming $S$. epidermidis strains ( $80 \%$ ) and additionally, each of strong biofilm formers were determined as multi drug resistant because of being resistant to 3 or more antimicrobial classes (Magiorakos et al., 2012) (Table 1). Moreover, one of these multi drug resistant strains which was found as resistant to maximum amount of different antimicrobial classes (7) was also observed as maximum biofilm forming strain among all the other $S$. epidermidis strains.

In parallel with our findings, Ponnusamy et al. (2012) demonstrate that, strong biofilm producers are more resistant to antimicrobial agents than weak biofilm producing ones. Similar with this; Kwon et al. (2008) also suggest that, multi drug resistant clinical isolates of $S$. aureus have a greater capability of developing biofilms on medical devices. In addition, according to an another study carried out on antibiotic resistance of exopolysaccharide forming $S$. epidermidis strains from orthopedic implant infections, similar to our results; up to $80 \%$ of all isolates were found as $\beta$-lactam resistant (Arciola et al., 2005).

All these findings show that, strong biofilm forming S. epidermidis strains display increased antimicrobial resistance and the reason of this is supposed to be related with genetic modifications occurring in the matrix of a biofilm. That is, our findings are in agreement with those of other studies suggesting; biofilm formation plays a huge role in gaining new virulence factors for any kind of microorganism (Donlan, 2001; Parsek and Singh, 2003; Subramanian et al., 2012).

To sum up, according to our results; samples of blood and wound were the most $S$. epidermidis isolated clinical materials and cardiothoracic surgery was the most $S$. epidermidis isolated service unit (Figures 1,2). Similar to this; strong biofilm forming S. epidermidis strains were also isolated from sample of blood among different clinical materials and from service of cardiovascular surgery among different service units in highest frequency (Figures 1,2). On the other hand, vancomycin was the most efficient antibiotic against all $S$. epidermidis strains whereas efficiency of $\beta$-lactam antibiotics all together (P, OX, AMC) was determined as $35 \%$ (Table 1). Additionally, each of strong biofilm forming $S$. epidermidis strains were multi drug resistant and high amount of them were resistant to all $\beta$-lactam antibiotics (P, OX, AMC) used in this study (Table 1). Furthermore, one of these multi drug resistant strains which was found to display resistance to maximum amount of different antimicrobial classes was also observed as maximum biofilm forming strain among all the other S. epidermidis strains.

\section{Conclusion}

This study shows that, a commensal skin colonizer may easily turn in to an important pathogen. Therefore, as well as preventing medical device using patients from extrinsic $S$. epidermidis contaminations, taking precautions against patient's own skin during surgical operations is also extremely necessary in order to prevent $S$. epidermidis related nosocomial infections. Otherwise, once $S$. epidermidis spreads to external surface of any bloodcontacting medical device during the settlement of it by surgical operation, colonization and biofilm formation of $S$. epidermidis on device's surface may come true and this may be the result of every reoccurring, persistent infections. Additionally, inside the matrix of biofilms, some genetic modifications may occur and turn these commensal microorganisms into multi drug resistant pathogens. As a result, surgeons must strictly take precautions against both extrinsic S. epidermidis contaminations and patient's own skin during surgical operations.

\section{References}

Al FD, Akça G, Sipahi B, Sultan N (2005) Kan Örneklerinden Soyutlanan Stafilokok Suslarinin Antibiyotiklere Direnç Durumlari. ANKEM 19(1):14-16.

Arciola CR, Campoccia D, Gamberini S, Donati ME, Pirini V, Visai L, Speziale P, Montanaro L (2005) Antibiotic resistance in exopolysaccharide-forming Staphylococcus epidermidis clinical isolates from orthopaedic implant infections. Biomaterials 26(33):6530-6535.

Arciola CR, Campoccia D, Speziale P, Montanaro L, Costerton JW (2012) Biofilm formation in Staphylococcus implant infections. A review of molecular mechanisms and implications for biofilm-resistant materials. Biomaterials 33(26):5967-5982.

Aybar Y, Ozaras R, Besirli K, Engin E, Karabulut E, Salihoglu T, Mete B, Tabak F, Mert A, Tahan G, Yilmaz MH, Ozturk R (2012) Efficacy of tigecycline and vancomycin in experimental catheter-related Staphylococcus epidermidis infec- 
tion: microbiological and electron microscopic analysis of biofilm. Int J Antimicrob Agents 39(4):338-342.

Baillie GS, Douglas LJ (2000) Matrix polymers of Candida biofilms and their possible role in biofilm resistance to antifungal agents. J Antimicrob Chemother 46:397-403.

Chaieb K, Abbassi MS, Touati A, Hassen AB, Mahdouani K, Bakhrouf A (2005) Molecular characterization of Staphyloccus epidermidis isolated from biomaterials in a dialysis service. Ann Microbiol 55(4):307-312.

Costerton JW (1999) Introduction to biofilm. Int J Antimicrob Agents 11:217-221.

Costerton JW, Lewandowski Z, Caldwell DE, Korber DR, Lappin-Scott HM (1995) Microbial biofilms. Annu Rev Microbiol 49:711-745.

de Araujo GL, Coelho LR, de Carvalho CB, Maciel RM, Coronado AZ, Rozebaum R, Ferreira-Carvalho BT, Figueiredo AMS, Teixera LA (2006) Commensal isolates of methicillin-resistant Staphylococcus epidermidis are also well equipped to produce biofilm on polystyrene surfaces. J Antimicrob Chemother 57:855-864.

Djeribi R, Bouchloukh W, Jouenne T, Menaa B (2012) Characterization of bacterial biofilms formed on urinary catheters. Am J Infect Control 40(9):854-859.

Donlan RM (2001) Biofilm formation: a clinically relevant microbiological process. Clin Infect Dis 33:1387-1392.

Fux CA, Stoodley P, Hall-Stoodley L, Costerton JW (2003) Bacterial biofilms: a diagnostic and therapeutic challenge. Expert Rev Anti Infect Ther 1:667-683.

Goldman M, Delage G, Beauregard P, Pruneau-Fortier D, Ismaïl J, Robillard P (2001) A fatal case of transfusion-transmitted Staphylococcus epidermidis sepsis. Transfusion 41:10751076.

Guiot HF, Visser LG, Barge RM, Bosboom RM, van de Klundert JA (1994) Fatal meningitis due to catheter-related Staphylococcus epidermidis bacteraemia in a granulocytopenic patient without predisposing trauma. Eur J Clin Microbiol Infect Dis 13(9):772-773.

Holt JG, Krieg NR, Sneath PHA, Staley JT, Williams ST (Editors) (1994) Bergey's Manual of Determinative Bacteriology, 9th edn The Williams \& Wilkins Co, Baltimore.

Juárez-Verdayes, MA, Reyes-López MA, Cancino-Díaz ME, Muñoz-Salas S, Rodríguez-Martínez S, de la Serna FJ, Hernández-Rodríguez $\mathrm{CH}$, Cancino-Díaz JC (2006) Isolation, vancomycin resistance and biofilm production of Staphylococcus epidermidis from patients with conjunctivitis, corneal ulcers, and endophthalmitis. Rev Latinoam Microbiol 48(3-4):238-246.

Kaplan JB, Ragunath C, Velliyagounder K, Fine DH, Ramasubbu N (2004) Enzymatic Detachment of Staphylococcus epidermidis Biofilms. Antimicrob Agents and Chemother 48(7):2633-2636.

Kumar CG Anand SK (1998) Significance of microbial biofilms in food industry: a review. Int J Food Microbiol 42:9-27.

Kwon AS, Park GC, Ryu SY, Lim DH, Lim DY, Choi CH, Park Y, Lim Y (2008) Higher biofilm formation in multidrugresistant clinical isolates of Staphylococcus aureus. Int J Antimicrob Agents 32(1):68-72.

Magiorakos AP, Srinivasan A, Carey RB, Carmeli Y, Falagas ME, Giske CG, Harbarth S, Hindler JF, Kahlmeter G, Olsson-Liljequist B, Paterson DL, Rice LB, Stelling J,
Struelens MJ, Vatopoulos A, Weber JT, Monnet DL (2012) Multidrug-resistant, extensively drug-resistant and pandrug-resistant bacteria: an international expert proposal for interim standard definitions for acquired resistance. Clin Microbiol Infect 18:268-281.

McCann MT, Gilmore BF, Gorman SP (2008) Staphylococcus epidermidis device-related infections: pathogenesis and clinical management. J Pharm Pharmacol 60(12):1551-1571.

Michelim L, Lahude M, Araújo PR, Giovanaz DSH, Müller G, Delamare APL, Costa SOP, Echeverrigaray S (2005) Pathogenicity factors and antimicrobial resistance of Staphylococcus epidermidis associated with nosocomial infections occurring in intensive care units. Braz J Microbiol 36:17-23.

O'Toole GA (2011) Microtiter dish biofilm formation assay. J Vis Exp 30(47):2437.

Otto M (2009) Staphylococcus epidermidis - the 'accidental' pathogen. Nat Rev Microbiol 7:555-567.

Parsek MR, Singh PK (2003) Bacterial Biofilms: An Emerging Link to Disease Pathogenesis. Annu Rev Microbiol 57:677701.

Ponnusamy P, Natarajan V, Sevanan M (2012) In vitro biofilm formation by uropathogenic Escherichia coli and their antimicrobial susceptibility pattern. Asian Pac J Trop Dis 5(3):210-213

Poutsiaka DD, Price LL, Ucuzian A, Chan GW, Miller KB, Snydman DR (2007) Blood stream infection after hematopoietic stem cell transplantation is associated with increased mortality. Bone Marrow Transplant 40:63-70.

Sheikh AF, Mehdinejad M (2012) Identification and determination of coagulase-negative Staphylococci species and antimicrobial susceptibility pattern of isolates from clinical specimens. Afr J Microbiol Res 6(8):1669-1674.

Shiro H, Muller E, Gutierrez N, Boisot S, Grout M, Tosteson TD, Goldmann D, Pier GB (1994) Transposon mutants of Staphylococcus epidermidis deficient in elaboration of capsular polysaccharide/adhesin and slime are avirulent in a rabbit model of endocarditis. J Infect Dis 169(5):1042-1049

Subramanian P, Shanmugam N, Sivaraman U, Kumar S, Selvaraj S (2012) Antibiotic resistance pattern of biofilm-forming uropathogens isolated from catheterised patients in Pondicherry, India. Australas Med J 5(7):344-348.

Tunney MM, Patrick S, Gorman SP, Nixon JR, Anderson N, Davis RI, Hanna D, Ramage G (1998) Improved detection of infection in hip replacements: a currently underestimated problem. J Bone Joint Surg 80B:568-572.

Villari P, Sarnataro C, Iacuzio L (2000) Molecular Epidemiology of Staphylococcus epidermidis in a Neonatal Intensive Care Unit over a Three-Year Period. J Clin Microbiol 38(5):1740-1746.

Vuong C, Otto M (2002) Staphylococcus epidermidis infections. Microbes Infect 4(4):481-489.

Xu LC, Siedlecki CA (2012) Submicron-textured biomaterial surface reduces staphylococcal bacterial adhesion and biofilm formation. Acta Biomater 8(1):72-81.

Ziebuhr W, Hennig S, Eckart M, Kränzler H, Batzilla C, Kozitskaya S (2006) Nosocomial infections by Staphylococcus epidermidis: how a commensal bacterium turns into a pathogen. Int J Antimicrob Agents 28:14-20.

All the content of the journal, except where otherwise noted, is licensed under a Creative Commons License CC BY-NC. 